12

\title{
Конический зеркальный энергоанализатор
}

\author{
(ㄱ) Л.А. Баранова
}

Физико-технический институт им. А.Ф. Иофрфе РАН,

194021 Санкт-Петербург, Россия

e-mail: baranova@mail.ioffe.ru

(Поступило в Редакцию 8 ноября 2017 г.)

Проведено исследование электронно-оптических свойств осесимметричного зеркального энергоанализатора, внутренний электрод которого представляет собой цилиндр, а внешний образован двумя идентичными конусами, примыкающими друг к другу своими основаниями. Показано, что светосила и разрешающая способность такого анализатора существенно превосходят аналогичные параметры традиционно используемого цилиндрического зеркала. Выполнение внутреннего электрода из трех цилиндров разного диаметра, находящихся под одним потенциалом, позволяет добиться дальнейшего улучшения электронно-оптических характеристик конического анализатора. При малых углах раствора пучка разрешение конического анализатора вдвое превосходит разрешение цилиндрического зеркала, при возрастании угла раствора разница в разрешении достигает порядка величины.

DOI: $10.21883 /$ JTF.2018.07.46184.2549

\section{Введение}

В научных исследованиях и в промышленности широкое применение получил цилиндрический зеркальный энергоанализатор. Достоинствами цилиндрического зеркала являются простота конструкции, высокая разрешающая способность, обусловленная наличием фокусировки второго порядка по меридиональному углу, и высокая светосила, обусловленная осевой симметрией анализатора [1-3]. В работах [4,5] предложен и испытан осесимметричный зеркальный энергоанализатор, светосила и разрешающая способность которого значительно превосходят аналогичные параметры цилиндрического зеркала. Существенным недостатком этого прибора является сложная форма электродов, описываемая уравнением $\ln r-\left(r^{2} / 2-z^{2}\right)=$ const. B [6] показано, что столь же высокие светосила и разрешение достигаются в цилиндрическом зеркальном анализаторе, если его внешний электрод разрезан на три части, потенциалы на которых можно регулировать независимо. В [7] показано, что дальнейшего улучшения электронно-оптических характеристик цилиндрического зеркала можно добиться в конструкции, где оба электрода (как внешний, так и внутренний) выполнены дискретными.

В настоящей работе предложена и исследована путем компьютерного моделирования еще одна конструкция осесимметричного зеркального анализатора, параметры которого не уступают параметрам анализаторов, описанных в [6,7]. Внешний электрод исследованной конструкции выполнен из конических электродов, внутренний образован одним или тремя цилиндрами.

\section{Конструкция конического зеркального анализатора}

Исследованы две модификации конического анализатора, слегка различающиеся строением внутреннего электрода. Внешний электрод в обеих модификациях образован двумя идентичными усеченными конусами, соприкасающимися своими основаниями. Внутренний электрод в первой модификации представляет собой сплошной цилиндр, во второй - образован тремя цилиндрами, причем радиусы двух крайних цилиндров одинаковы, а радиус среднего цилиндра несколько больше

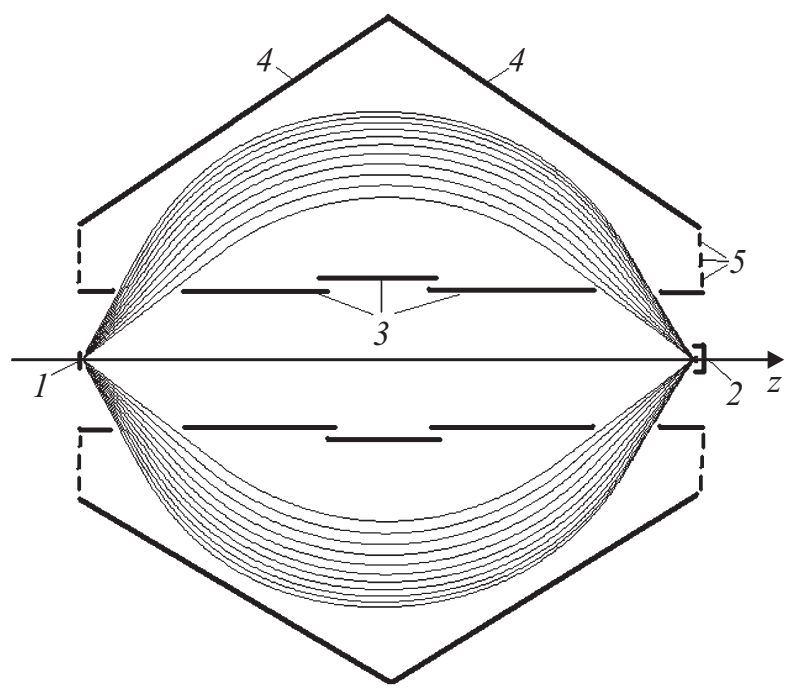

Рис. 1. Схематическое изображение конического зеркального анализатора: 1 - исследуемый образец, 2 - детектор, 3 - внутренний цилиндрический электрод, $4-$ внешний конический электрод, 5 - электроды, компенсирующие краевое поле. 
радиуса крайних. Потенциал внутреннего электрода во всех случаях равен нулю.

Схематическое изображение конического зеркального анализатора представлено на рис. 1 (вторая модификация). Источник заряженных частиц и детектор расположены на оси симметрии анализатора - режим фокусировки „ось-ось“. На каждом из торцов анализатора с целью компенсации краевого поля помещены кольцевые электроды, потенциалы на которых определяются путем расчета распределения потенциала в неусеченных конуcax. Поскольку на торцах расстояние между внешним и внутренним электродами в коническом анализаторе существенно меньше, чем в цилиндрическом, проблема компенсации краевого поля здесь стоит менее остро.

\section{Определение оптимальных геометрических параметров конического анализатора}

Электронно-оптические свойства конического зеркального анализатора исследовались численно с помощью программы CPO3D [8], позволяющей с высокой точностью рассчитывать распределение потенциала и траектории движения заряженных частиц в трехмерных электростатических системах. Было рассчитано несколько вариантов конического анализатора, различающихся радиусом основания конусов, углом при вершине и положением плоскости, в которой конусы соприкасаются, а также длиной и радиусом центральной части внутреннего электрода. Для каждого варианта конструкции определялся потенциал на внешнем электроде, обеспечивающий фокусировку второго порядка на оси анализатора. Рис. 2 иллюстрирует поиск условий фокусировки второго порядка, на нем представлена зависимость координаты точки пересечения траектории

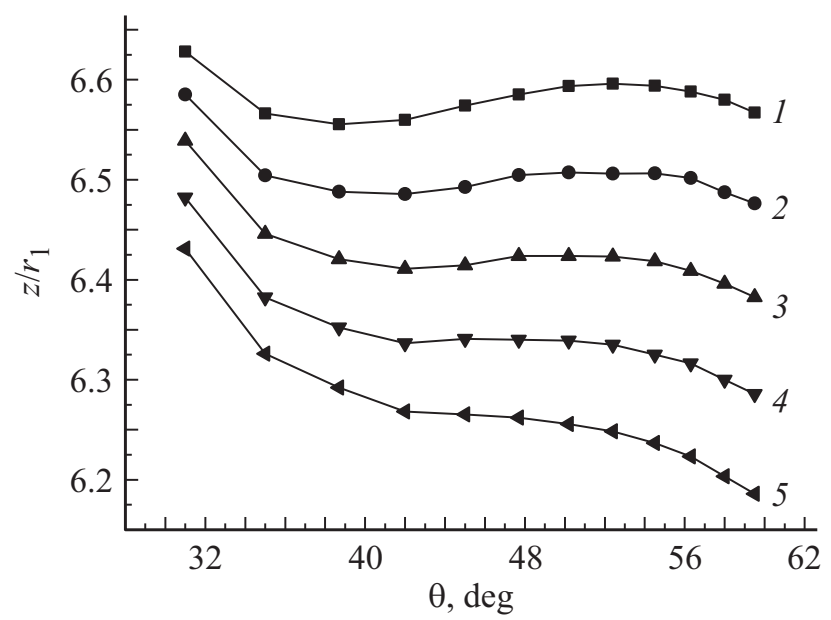

Рис. 2. Координата точки пересечения траектории заряженной частицы с осью $z$ в зависимости от угла входа заряженных частиц $\theta$ при различных значениях потенциала на внешнем электроде: $1-\mathrm{eV} / E=0.66,2-0.67,3-0.68,4-0.69$, $5-0.7$. заряженной частицы с осью $z$ от угла $\theta$ влета частицы в анализатор. Параметром семейства кривых является значение потенциала на внешнем электроде $V$, отнесенное к энергии частиц, выраженной в электрон-вольтах $(e V / E)$. Кривая 4 соответствует тому случаю, когда производная $d^{2} z / d \theta^{2}$ обращается в нуль, т. е. достигается фокусировка второго порядка. На основании полученных данных определялись базовые электронно-оптические характеристики каждой из исследованных конструкций в режиме фокусировки второго порядка.

Проведенное исследование позволило найти оптимальные геометрические параметры конического анализатора, обеспечивающие наилучшее разрешение и светосилу. Строение внешнего электрода одинаково в обеих модификациях. Радиус общего основания конусов $r_{c 1}$, отнесенный к радиусу внутреннего цилиндра $r_{1}$, равен $r_{c 1} / r_{1}=3.3$. Конусы соприкасаются в плоскости, находящейся на расстоянии $z / r_{1}=3.3$ от источника заряженных частиц. Радиус сечения конуса плоскостью, проходящей через источник заряженных частиц, равен $r_{c 2} / r_{1}=1.7$. Как уже указывалось выше, внутренний электрод первой модификации представляет собой сплошной цилиндр радиуса $r_{1}$. Во второй модификации внутренний электрод образован тремя цилиндрами. Отношение радиуса центрального цилиндра к радиусу крайних составляет $r_{2} / r_{1}=1.15$, длина центрального цилиндра равна радиусу $r_{1}$, расстояние от его центра до источника заряженных частиц составляет $3.3 r_{1}$. Данные, представленные на рис. 2, рассчитаны именно для оптимальной конструкции конического анализатора.

\section{Электронно-оптические характеристики конического анализатора}

Рис. 3 иллюстрирует эквипотенциальные линии и траектории заряженных частиц в коническом анализаторе (вторая модификация), рассчитанные программой CPO3D. Угол раствора пучка заряженных частиц составляет $\Delta \theta=60^{\circ}-35^{\circ}=25^{\circ}$. Как видно из рис. 3, даже при таком экстремально большом угле раствора сохраняется острая фокусировка пучка частиц на оси анализатора. Режим работы, представленный на рис. 3 , соответствует кривой 2 на рис. $2(e V / E=0.67)$. В этом режиме достигается высокая светосила 15\% при сравнительно небольшом разрешении $0.7 \%$. На оси анализатора расположен кроссовер (наименьшее сечение) пучка, в то время как параксиальное изображение источника заряженных частиц располагается дальше по ходу пучка. В режимах с большим значением потенциала на внешнем электроде (кривые 3,4) можно добиться лучшего разрешения, уменьшая угол захвата пучка, т.е. светосилу. Наилучшее разрешение достигается в режиме, соответствующем кривой $4(e V / E=0.69)$, когда параксиальное изображение источника совмещено с осью анализатора, при этом, однако, угол захвата пучка будет минимален. При разрешении в 0.04\% светосила 


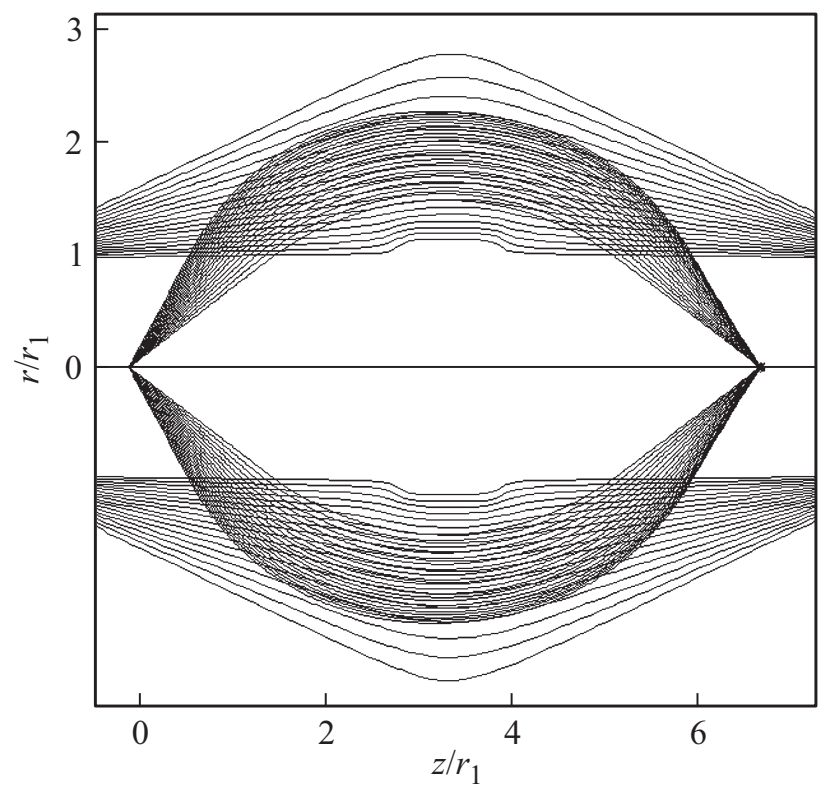

Рис. 3. Эквипотенциальные линии и траектории заряженных частиц в коническом зеркальном анализаторе.

Разрешение $\rho$ трех типов зеркального энергоанализатора в зависимости от угла раствора $\Delta \theta^{\circ}$ входящего пучка

\begin{tabular}{c|c|c|c}
\hline $\begin{array}{c}\text { Угол } \\
\text { раствора } \\
\text { пучка } \Delta \theta\end{array}$ & $\begin{array}{c}\text { Цилиндрическое } \\
\text { зеркало. } \\
\text { Разрешение } \rho\end{array}$ & $\begin{array}{c}\text { Конический } \\
\text { анализатор 1. } \\
\text { Разрешение } \rho\end{array}$ & $\begin{array}{c}\text { Конический } \\
\text { анализатор 2. } \\
\text { Разрешение } \rho\end{array}$ \\
\hline 7 & 0.001 & 0.0008 & 0.0004 \\
11 & 0.008 & 0.002 & 0.0015 \\
17 & 0.03 & 0.004 & 0.003 \\
23 & 0.05 & 0.006 & 0.004 \\
27 & 0.07 & 0.012 & 0.009 \\
29 & - & 0.015 & 0.011
\end{tabular}

анализатора составит 4.7\%. Таким образом, выбор режима работы (потенциала на внешнем электроде) зависит от требований к соотношению разрешения анализатора и его светосилы. Следует учитывать, что при изменении потенциала на внешнем электроде положение кроссовера пучка сдвигается вдоль оси $z$ и соответственно меняется оптимальное положение детектора.

В таблице приведены значения разрешения конического анализатора в зависимости от угла раствора пучка заряженных частиц (т.е. в зависимости от его светосилы). Здесь же для сравнения даны соответствующие значения разрешения стандартного цилиндрического зеркала. При углах раствора пучка, не превышающих $7^{\circ}$, разрешение конического анализатора первой модификации и цилиндрического зеркала примерно одного порядка. Разрешение конического анализатора второй модификации вдвое лучше. При возрастании угла раствора разница в качестве фокусировки стремительно возрастает. При углах раствора пучка, превышающих $17^{\circ}$, разрешение конического анализатора на порядок лучше разрешения цилиндрического зеркала.

\section{Заключение}

На основании компьютерного моделирования в работе исследован конический зеркальный энергоанализатор заряженных частиц. Найдены соотношения основных геометрических параметров, обеспечивающие наилучшие разрешение и светосилу анализатора. Показано, что основные электронно-оптические характеристики исследованного анализатора существенно превосходят аналогичные параметры цилиндрического зеркала, традиционно используемого в электронной спектроскопии. При одинаковом угле раствора пучка заряженных частиц на входе в анализатор, равном $7^{\circ}$, разрешение конического анализатора второй модификации составляет $0.04 \%$ и вдвое превосходит разрешение цилиндрического зеркала. С увеличением угла раствора пучка разрешение конического анализатора деградирует гораздо медленнее, чем разрешение цилиндрического зеркала, что позволяет работать с большой светосилой при удовлетворительном разрешении. Так, при одинаковом разрешении 0.7\% светосила конического анализатора почти в три раза выше светосилы цилиндрического зеркала и составляет $15 \%$ от полной сферы.

\section{Список литературы}

[1] Blauth E. // Zs. Phys. 1957. Vol. 147. P. 228-231.

[2] Зашквара В.В., Корсунский М.И., Космачев О.С. // ЖТФ. 1966. T. 36. C. $132-138$.

[3] АЯанасьев В.П., Явор С.Я. Электростатические энергоанализаторы для пучков заряженных частиц. М.: Наука, 1978. $224 \mathrm{c}$.

[4] Siegbanh K., Golikov Yu., Kholine N. A high resolution and large transmission spectrometer. Tecnikum, Institute of Technology, Uppsala University, 1996.

[5] Голиков Ю.К., Кольцов С.Н., Холин Н.А. // Изв. АН. Сер. физ. 1998. Т. 62. № 3. С. 555.

[6] Баранова Л.А. // ЖТФ. 2013. Т. 83. Вып. 4. С. 149-151. [Baranova L.A. // Tech. Phys. 2013. Vol. 58. N 4. P. 615-617.]

[7] Баранова Л.А. // ЖТФ. 2017. Т. 87. Вып. 3. С. 458-461. [Baranova L.A. // Tech. Phys. 2017. Vol. 62. N 3. P. 480-483.]

[8] CPO programs, available from www.electronoptic.com 